\title{
Variance-based salt body reconstruction
}

Oleg O. Ovcharenko

Vladimir V. Kazei

Daniel Peter

Tariq Alkhalifah

King Abdullah University of Science and Technology (KAUST), Division of Physical Sciences and Engineering, Thuwal 23955-6900, Saudi Arabia

\section{Summary}

Seismic inversions of salt bodies are challenging when updating velocity models based on Born approximationinspired gradient methods. We propose a variance-based method for velocity model reconstruction in regions complicated by massive salt bodies. The novel idea lies in retrieving useful information from simultaneous updates corresponding to different single frequencies. Instead of the commonly used averaging of single-iteration monofrequency gradients, our algorithm iteratively reconstructs salt bodies in an outer loop based on updates from a set of multiple frequencies after a few iterations of full-waveform inversion. The variance among these updates is used to identify areas where considerable cycle-skipping occurs. In such areas, we update velocities by interpolating maximum velocities within a certain region. The result of several recursive interpolations is later used as a new starting model to improve results of conventional full-waveform inversion. An application on part of the BP 2004 model highlights the evolution of the proposed approach and demonstrates its effectiveness.

Topics: Full Waveform Seismic Inversion, Seismic Imaging - Theory, Velocity and Seismic Velocity Parameter Estimation - Theory

Main objectives: Introduce a computationally inexpensive semi-automated method for inverting salt bodies.

New aspects covered: We consider updates from different single frequencies through several iterations instead of commonly used single-iteration single-frequency gradients averaging. We propose a computationally inexpensive way to build an initial model for full-waveform inversion from relatively high frequencies. 


\section{Introduction}

Seismic inversions of salt bodies are one of the major challenges in full waveform inversion (FWI). In acoustic inversions, the salt-induced challenge is primarily plagued with cycle-skipping phenomena (Bunks et al., 1995). Cycle-skipping occurs when a phase shift on the wavepath from the source to the receiver is larger than half of the dominant period of the signal, and thus, depends on both the wavepath and the wavelength of the signal. Signals corresponding to long wavepaths in which energy travels through deeper parts of the model are more prone to cycle-skipping. This guides FWI to a local minima of the objective function and prevents further model improvements, especially in deep explorations. Gradual offset increase (Brossier et al., 2009) or various misfit functions (Choi and Alkhalifah, 2015, e.g.) can help mitigate the problem in the data domain. Gradient conditioning can serve the same purpose through scattering angle-based filters (Alkhalifah, 2016; Kazei et al., 2016), image-guided inversion (Ma et al., 2012) or gradient optimization (Wu and Alkhalifah, 2016).

Nowadays, in high-contrast salt regions the preferred approach is utilizing imaging along with salt flooding to obtain a reasonable velocity model for FWI. Such a flooding process requires a considerable amount of user intervention including top of the salt picking on a migrated image, which is often prone to user errors. Several approaches to solve the problem have been proposed. Most of them are relatively expensive and rely on either additional regularization terms (Esser et al., 2015) or explicit assumptions about the model velocity distribution (Kadu et al., 2016).

Here, we attempt to locate areas in which updates are determined to correspond to cycle-skipped wavepaths and update them differently until the background model is mature enough for them to be handled. We compare simultaneous updates corresponding to different single frequencies using a variance criteria. This allows us to identify salt regions in the current model and to update the deep parts of the velocity model using a flooding technique. In the following, we present the method of our variance-based salt body reconstruction technique and demonstrate its applicability in a synthetic benchmark of a massive salt body model.

\section{Method}

To enhance the signal-to-noise ratio, FWI often uses several bands of frequencies (Brossier et al., 2009) in a multi-scale approach (Bunks et al., 1995). The common approach in frequency-domain FWI is stacking multiple misfit functionals for single frequencies. This gives rise to summing together all gradients from different frequencies at each iteration. In this work, we exploit the fact that notable cycle-skipping artifacts often occur in presence of a large salt body. We illustrate the problem in a synthetic FWI application to a resampled part of the BP 2004 model, shown in Figure 1(a). As a starting model for FWI, a linearly increasing with depth velocity is chosen. Conventional multi-scale FWI gets stuck in a local minimum of the misfit functional (Figure 1(b)). Instead of stacking multiple misfit functionals, we propose to minimize misfits from different frequencies independently with an L-BFGS optimization scheme and subsequently examine and compare updates in a separate step to identify salt body areas. Figure 2 illustrates cycle-skipping artifacts in the presence of a large salt body at different dominant frequencies. Locations of these artifacts shift for different frequencies, thus regions with high variance among different updates highlight areas where the velocity model update is less reliable. We iteratively locate these areas based on a variance criteria and process updates differently inside them using a flooding technique.

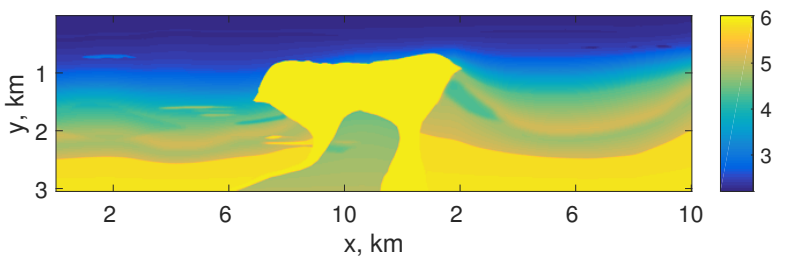

(a)

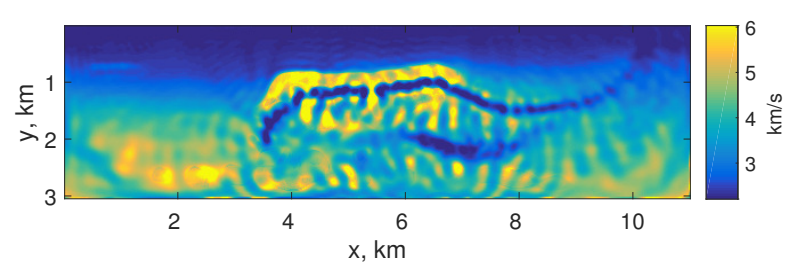

(b)

Figure 1 Conventional multi-scale FWI. (a) True model - resampled crop from the 2004 BP VelocityAnalysis Benchmark $(N X=550, N Z=152, \Delta x=\Delta z=20 \mathrm{~m})$. (b) Final inversion result using sequential frequencies from 4 to $7 \mathrm{~Hz}$ (starting from an initial velocity model with a constant vertical gradient $\left.v=v_{0}+\alpha z\right)$

The processing workflow consists of 5 principal steps: 


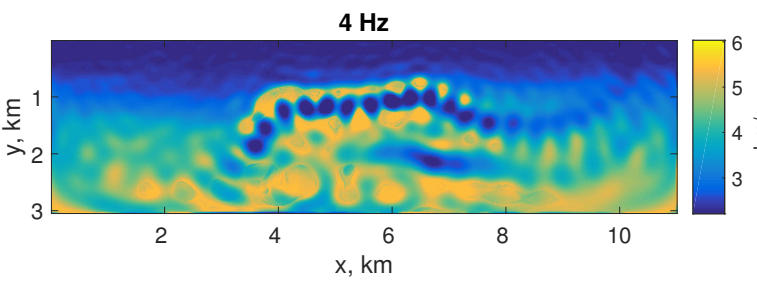

(a)

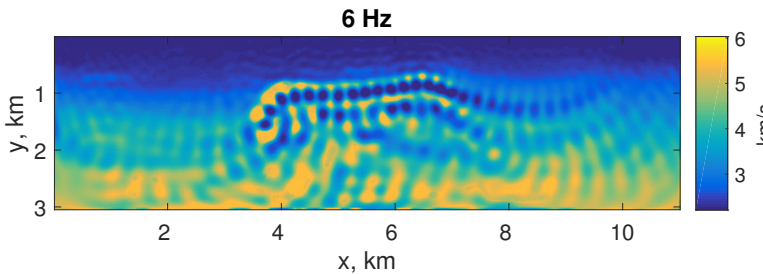

(c)

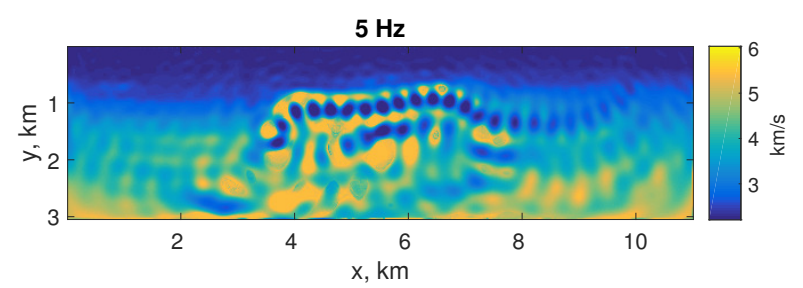

(b)

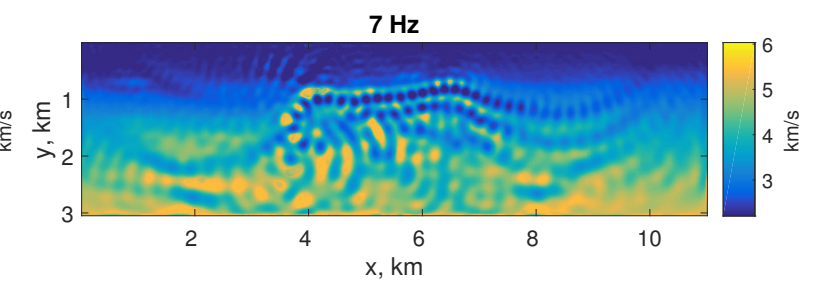

(d)

Figure 2 Conventional FWI at single frequencies. (a-d) Cycle-skipping artifacts (dark blue spots) are noticeable in all inversion results after 50 iterations in range $4-7 \mathrm{~Hz}$. Areas illuminated by short wavepaths (primarily near-surface) exhibit less variations.

1. Collect several independent FWI updates at single frequencies. It is well known that migration images from high frequencies look different even if the initial model is close to the real one. However, by considering updates after several FWI iterations, shallow areas where cycle skipping rarely happens exhibit nearly identical velocities with low variance. As seen in Figure 2(a)-(d), the top of the salt body becomes well distinguishable and almost invariant.

2. Stack and average updates. Model updates after a few iterations of FWI at single frequencies provide similar content. In a subsequent step, they are summed up and averaged as shown in Figure 3(a). As a result of this averaging, cycle-skipping artifacts are reduced.

3. Build a variance map between updates. High-variance areas seen in Figure 3(b) often appear near or within salt bodies and where cycle-skipping is most prominent. Both issues should be resolved, so processing of such model regions should reduce harmful effects of cycle-skipping and highlight the salt body. These high-variance areas shape a "variance mask" as shown in Figure 4(a). These masked areas should be flooded gradually with salt in case they are beneath and in the vicinity of a known salt region.

4. Detect unalterable high-velocity regions. In general, the top of a salt body is well resolved at all reasonable frequencies. Hence, as a result of stacking, areas which coincide through most images are getting enhanced. These areas with highest velocity values on the stacked image shape a "salt mask" and will be marked as part of a salt body, also shown in Figure 4(a).

5. Apply area defragmentation and flooding. Identifying two separate masks which cover areas with unalterable high-velocity and high-variance values will likely lead to strongly fragmented areas due to arbitrary shape and distribution of selected features. An additional processing step is thus required to reduce the fragmentation of selected areas, creating a connected model space as shown in Figure 4(b). We apply first a non-stationary smoothing filter, and then crop off the edges. Finally, we perform flooding within areas under a "salt mask" covered by a "variance mask". The maximum velocity value found in a radius related to the local wavelength is used as a flooding constant. This improves the robustness of the workflow by avoiding the need to create domains with artificial salt values, like single level set methods would do (Kadu et al., 2016).

The goal of the proposed workflow is to create a new velocity model with partial reconstruction of the salt body. This new model can then be used as a starting model in a successive, conventional multi-scale FWI. The workflow can naturally be included in an iterative scheme to update further salt body areas.

\section{Results}

Acoustic FWI was performed sequentially for frequencies from 4 to $7 \mathrm{~Hz}$ with steps of $1 \mathrm{~Hz}$. Conventional FWI fails when using a one-dimensional starting model in our setup Figure 5(b). In contrast, the proposed iterative flooding approach allows to reconstruct the main salt body features Figure 5(c) at 4-7 


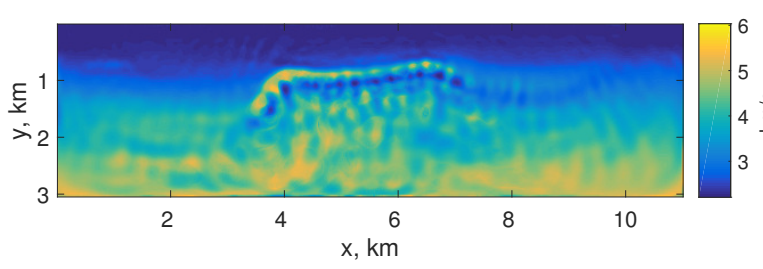

(a)

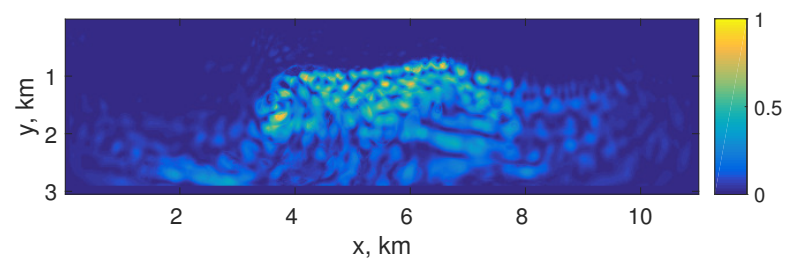

(b)

Figure 3 Update average and variance. (a) Average velocity values of corresponding model updates after first 10 iterations of FWI at 4-7 Hz. (b) Normalized variance between corresponding updates.

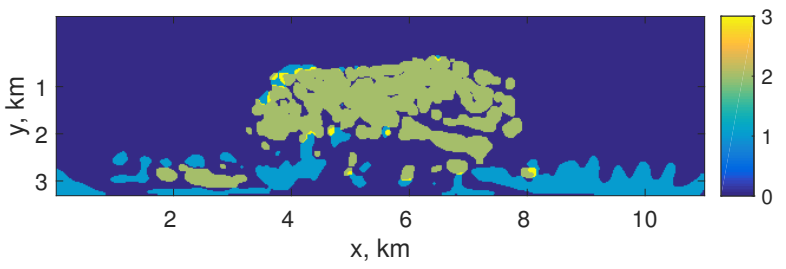

(a)

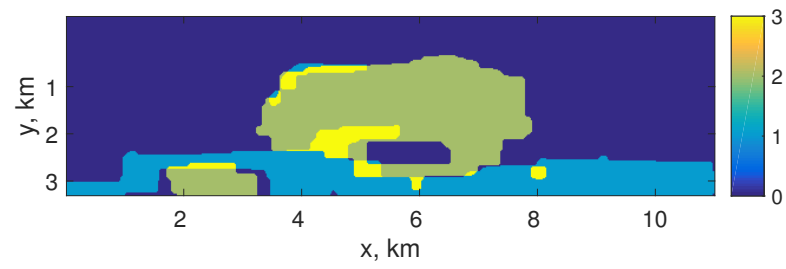

(b)

Figure 4 Detection of salt body masks. (a) Fragmented masks and (b) Defragmented masks in order to get smoother fill. Color values used are 1 for "salt mask", 2 for "variance mask" and 3 for overlap area.

$\mathrm{Hz}$ and used as a starting model leads FWI to the much better final result (Figure 5(d)). In this example flooding was performed recursively 4 times after 10 iterations each.

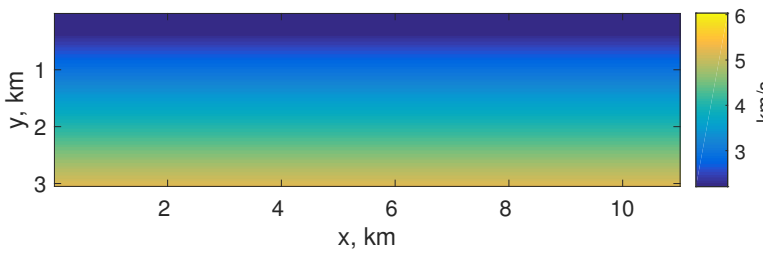

(a)

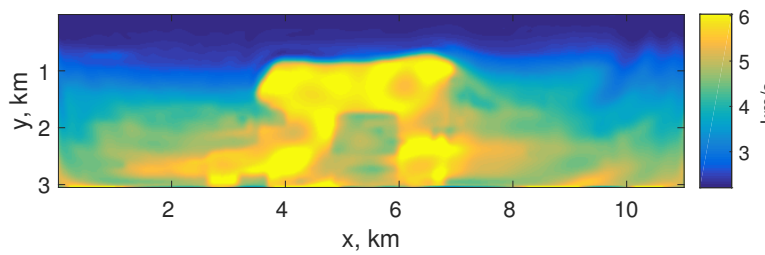

(c)

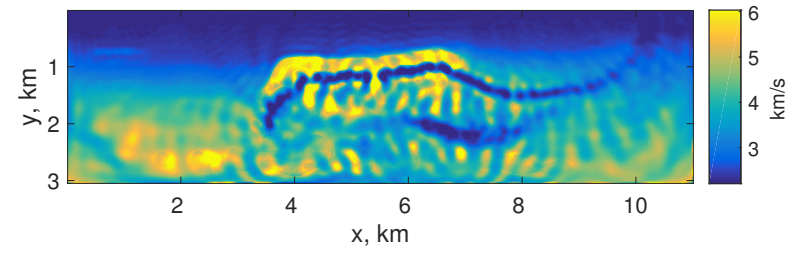

(b)

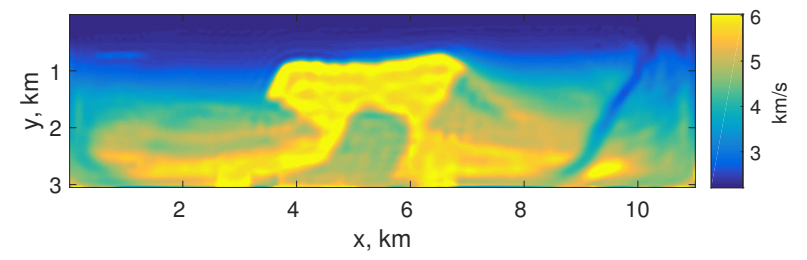

(d)

Figure 5 FWI with variance-based salt body reconstruction. Initial models: (a) one-dimensional velocity model with constant gradient; (c) variance-based salt body reconstruction model after 5 sequential floodings with $10 \mathrm{FWI}$ iterations each. Resulting models: velocity model after 50 iterations of conventional FWI at 4-7 Hz, starting (b) from gradient and (d) flooded initial model, shown in (a) and (c) respectively.

The computational cost of the approach is slightly higher when included in FWI. Nevertheless, the increase is overall negligible as soon as higher frequencies are taken into account. High-frequency inversions require most of the computational time, with a high vulnerability of the FWI scheme to fail in case of an improper model parameter selection. We hope to mitigate this problem by providing a fully automated parameter selection procedure in the future. Furthermore to improve signal-to-noise ratios in images for more realistic applications, narrow bands of frequencies can be used instead of single frequencies in the presence of noise.

\section{Discussion}

The proposed variance-based salt body reconstruction technique extends conventional FWI schemes. It only takes model updates and frequencies at which conventional FWI would be done. The technique 
then provides an alternative, flooded model, update. Thus, the proposed workflow can be easily embedded into any conventional FWI routine. However, apart from a set of standard parameters involved in frequency-domain FWI, additional tuning parameters appear in the proposed workflow:

1. Number of FWI iterations before flooding: This number usually depends on the optimization algorithm that is used. Unfortunately, salt bodies often cause strong multi-scattering which results in failure of computationally inexpensive (in frequency-domain) Gauss-Newton optimization methods (Métivier et al., 2013). On the other hand, L-BFGS and truncated Newton methods can handle strong multi-scattering at the cost of higher computational efforts. Several iterations of L-BFGS can potentially be replaced with a single iteration of truncated Newton optimization, with a better stopping criterion than currently used in this work.

2. Gap between single frequencies: This gap has to be sufficient to cause a shift of the locations of cycle-skipping artifacts, thus it should be related to the typical propagation times in the background model and minimum frequency available in the data.

3. Number of repeated flooding iterations: Ideally the process should stop when there are no more high-variance regions. However, our experiments show that after a few flooding cycles, the inversion stales and they are enough to build a sufficiently good initial model. Complementary, standard FWI exit conditions can be used to reach a final velocity model.

To fully automate these additional input parameters, further testing of the proposed flooding technique on different models using various minimization techniques is needed and will be left for future research.

\section{Conclusions}

We have shown that considering the variance between different single-frequency FWI updates can be a complementary source of information for salt body shape and content recovery. High variance values highlight regions either where model updates are unreliable or where cycle-skipping artifacts occur. We showed that additional processing of such areas using a salt flooding technique can be beneficial in exploration setups with large salt bodies. Furthermore, variance-based salt body reconstructions can be easily incorporated into existing FWI routines where it is common to use groups of frequencies in multi-scale inversions. Being properly tuned, the variance-based technique described in this work allows to overcome the lack of low-frequency seismic data and estimate the salt body shape and contrast well enough for further conventional multi-scale FWI usage.

\section{References}

Alkhalifah, T. [2016] Full-model wavenumber inversion: An emphasis on the appropriate wavenumber continuation. GEOPHYSICS, 81(3), R89-R98.

Brossier, R., Operto, S. and Virieux, J. [2009] Seismic imaging of complex onshore structures by 2D elastic frequency-domain full-waveform inversion. Geophysics, 74(6), WCC105-WCC118.

Bunks, C., Saleck, F.M., Zaleski, S. and Chavent, G. [1995] Multiscale seismic waveform inversion. Geophysics, 60(5), 1457-1473.

Choi, Y. and Alkhalifah, T. [2015] Unwrapped phase inversion with an exponential damping. Geophysics, 80(5), R251-R264.

Esser, E., Guasch, L., van Leeuwen, T., Aravkin, A.Y. and Herrmann, F.J. [2015] Automatic salt delineation?Wavefield Reconstruction Inversion with convex constraints. In: 2015 SEG Annual Meeting. Society of Exploration Geophysicists.

Kadu, A., Van Leeuwen, T. and Mulder, W. [2016] A parametric level-set approach for seismic fullwaveform inversion. In: SEG Technical Program Expanded Abstracts 2016, Society of Exploration Geophysicists, 1146-1150.

Kazei, V., Tessmer, E. and Alkhalifah, T. [2016] Scattering angle-based filtering via extension in velocity. In: SEG Technical Program Expanded Abstracts 2016, Society of Exploration Geophysicists, $1157-1162$.

Ma, Y., Hale, D., Gong, B. and Meng, Z.J. [2012] Image-guided sparse-model full waveform inversion. Geophysics, 77(4), R189-R198.

Métivier, L., Brossier, R., Virieux, J. and Operto, S. [2013] Full waveform inversion and the truncated Newton method. SIAM Journal on Scientific Computing, 35(2), B401-B437.

$\mathrm{Wu}, \mathrm{Z}$. and Alkhalifah, T. [2016] The optimized gradient method for full waveform inversion and its spectral implementation. Geophysical Journal International, 205(3), 1823-1831. 